\title{
SMOOTH PERTURBATIONS OF REGULAR DIRICHLET FORMS
}

\author{
PETER STOLLMANN
}

(Communicated by J. Marshall Ash)

\begin{abstract}
Given a regular Dirichlet form $\mathfrak{h}$, we prove that a measure $\mu$ is smooth iff the domain of $\mathfrak{h}+\mu$ is dense in the domain of $\mathfrak{h}$ with respect to the form norm. The latter condition is in turn equivalent to the convergence of $\mathfrak{h}+a \mu$ to $\mathfrak{h}$ as $a \downarrow 0$.
\end{abstract}

\section{INTRODUCTION AND PRELIMINARIES}

The study of Schrödinger operators of the form $-\Delta+\mu$, where $\mu$ is a measure, has attracted the attention of various authors in recent years; we only mention [1-8, 14].

If $\mu$ is a nonnegative measure that does not charge sets of zero capacity, one can define this operator by means of quadratic forms, as we will see in $\S 1$.

Smooth measures have turned out to be especially important, since one has an analogue for the Feynman-Kac formula for the semigroup generated by $-\Delta+\mu$ (see [9, Chapter 5; 15], where the perturbation of a Hunt process by additive functionals has been analyzed in detail).

The main result of this note, Theorem 2.2 , is a characterization of smooth measures in terms of very natural operator theoretic conditions; namely, $\mu$ is smooth iff $-\Delta+a \mu$ converge to $-\Delta$ in the strong resolvent sense as $a \downarrow 0$.

Let us now introduce the general framework of the present article. The monograph [9] is our standard reference for the following notions. (Nevertheless, we use the notation from [10] as far as forms are concerned.) We fix a locally compact $\sigma$-compact Hausdorff space $\mathbf{X}$ and a Radon measure $\mathbf{m}$ on $(\mathbf{X}, \mathfrak{B}$ ) (where $\mathfrak{B}$ denotes the Borel $\sigma$-algebra) such that $\operatorname{supp} \mathbf{m}=\mathbf{X}$. We assume that a regular Dirichlet form $\mathfrak{h}$ with domain $\mathbf{D}(\mathfrak{h})=\mathbf{D}$ on $\left(\mathbf{L}_{2}(\mathbf{X}, \mathbf{m}),(\cdot \mid \cdot)\right)$ is given, so that $\mathbf{D}$, equipped with the inner product $(\cdot \mid \cdot)_{\mathfrak{h}}:=(\mathfrak{h}+1)[\cdot, \cdot]=\mathfrak{h}[\cdot, \cdot]+(\cdot \mid \cdot)$, is a Hilbert space. Consequently, there exists a nonnegative self-adjoint operator $H$ in $L_{2}(\mathbf{X}, \mathbf{m})$ that corresponds to $h(H$ plays the role of $-\Delta)$.

We are mainly interested in the form sum $\mathfrak{h}+\mu$ that can be defined whenever $\mu$ is a measure that does not charge sets of zero capacity. This will be shown in $\S 1$ where we also give a necessary and sufficient condition for $\mu$ in order that $\mathfrak{h}+\mu$ be densely defined. The main result of the second section is the fact that

Received by the editors April 1, 1991.

1991 Mathematics Subject Classification. Primary 31C25; Secondary 35J10, 60J60, 60 J65.

Key words and phrases. Regular Dirichlet forms, smooth measures, Schrödinger operators. 
$\mu$ is smooth if and only if the domain $\mathbf{D}(\mathfrak{h}+\mu)$ is dense in $\left(\mathbf{D},(\cdot \mid \cdot)_{\mathfrak{h}}\right)$, which, in turn, is equivalent to $\mathfrak{h}+a \mu \rightarrow \mathfrak{h}$ in the strong resolvent sense for $a \downarrow 0$. In particular, this answers a question in [3] and relates the regular potentials of $[12,13,16]$ to smooth measures; namely, $V$ is regular if and only if $V d x$ is smooth. Some of the material in $\S \S 1$ and 2 is obtained by a straightforward generalization of results in [12].

\section{Measures that do NOT ChaRge SETS OF ZERO CAPACITY}

By $\operatorname{Cap}(A)=\operatorname{Cap}_{\mathfrak{h}}(A)$ we denote the capacity of a set $A \subset X$, which is defined according to $[9, \S 3.1]$. If $f$ is an element in $\mathbf{D}$ then there exist quasicontinuous modifications of $f$ [loc. cit.], the set of which will be denoted by $[f]_{q}$. The notation $f^{\sim}$ will be used for a quasi-continuous representative of $f$. In this section we shall consider the class

$\mathscr{M}_{0}:=\mathscr{M}_{0}(\mathfrak{h}):=\{\mu: \mathfrak{B} \rightarrow[0, \infty] ; \mu$ is a $\sigma$-additive measure such that

$$
\mu(B)=0 \text { for every } B \in \mathfrak{B} \text { with } \operatorname{Cap}(B)=0\}
$$

of measures that do not charge sets of zero capacity. It will turn out that " $\mu \epsilon$ $\mathscr{M}_{0}$ " is the adequate condition for the definition of $\mathfrak{h}+\mu$, if one is willing to consider nondensely defined forms.

Example 1. By $\mathfrak{h}=0$ a bounded regular Dirichlet form is given; $\operatorname{Cap}(B)=$ $\mathbf{m}(B)$ for every $B \in \mathfrak{B}$ and $\mathscr{M}_{0}=\{V \mathbf{m} ; V: \mathbf{X} \rightarrow[0, \infty], V$ measurable $\}$.

It is sometimes interesting to consider the above trivial example, however, the typical situation one should think of is

Example 2. $\mathbf{X}=\mathbb{R}^{\nu}, \mathbf{m}=d x$ the Lebesgue measure, $\mathbf{D}=W_{2}^{1}\left(\mathbb{R}^{\nu}\right)$, and $\mathfrak{h}[u, v]:=\int \nabla u \cdot \nabla \bar{v} d x$ define a regular Dirichlet form. For the special case $\nu=1, \operatorname{Cap}(B)>0$ for every $B \neq \varnothing$. Consequently,

$$
\mathscr{M}_{0}=\{\mu: \mathfrak{B} \rightarrow[0, \infty]: \mu \text { a measure }\} .
$$

1.1. Proposition. Let $\mu \in \mathscr{M}_{0}$. Then

$$
\mathbf{D}(\mu):=\left\{f \in \mathbf{D} ;[f]_{q} \subset \mathscr{L}_{2}(\mathbf{X}, \mu)\right\}, \quad \mu[f, g]:=\int f^{\sim}(x) \bar{g} \sim \mu(d x)
$$

defines a closed form on $\left(\mathbf{D},(\cdot \mid \cdot)_{\mathfrak{h}}\right)$.

Proof. Let $\left(f_{n}\right)$ be a sequence in $\mathbf{D}, f_{n} \rightarrow f$ in $\left(D,(\cdot \mid \cdot)_{\mathfrak{h}}\right)$, such that $\mu\left[f_{n}-f_{m}\right] \rightarrow 0$ for $n, m \rightarrow \infty$; from [9, Theorem 3.1.4] it follows that we can find a subsequence satisfying $f_{n_{k}}^{\sim} \rightarrow f^{\sim}$ q.e. Since $\mu \in \mathscr{M}_{0}$, this implies $f_{n_{k}}^{\sim} \rightarrow f^{\sim} \mu$-a.e. By assumption, $\left(f_{n_{k}}^{\sim}\right)$ is a Cauchy sequence in $\mathscr{L}_{2}(\mathbf{X}, \mu)$, which implies $f \in \mathscr{L}_{2}(\mathbf{X}, \mu), \mu\left[f_{n}-f\right] \rightarrow 0$.

As an immediate consequence, we have

1.2. Theorem. Let $\mu \in \mathscr{M}_{0}$. Then

$$
\mathbf{D}(\mathfrak{h}+\mu):=\mathbf{D}(\mu), \quad(\mathfrak{h}+\mu)[f, g]:=\mathfrak{h}[f, g]+\mu[f, g]
$$

for $f, g \in \mathbf{D}(\mathfrak{h}+\mu)$, defines a closed nonnegative form on $\left(L_{2}(\mathbf{X}, \mathbf{m}),(\cdot \mid \cdot)\right)$. 
Even though, in general, $\mathfrak{h}+\mu$ need not be densely defined we may follow Simon [11] and define the resolvent $\mathrm{R}(E, \mathfrak{h}+\mu)$ for each $E>0$.

Remark. In the situation of Example 2, with $G=\mathbb{R}^{\nu}$,

$$
\mathrm{R}(E, \mathfrak{h}+\mu)=\mathrm{R}_{\mu}^{G}(E),
$$

where the right-hand side is the "variational resolvent" of [5, Definition 2.3]; see also [5, Proposition 2.1].

Following the ideas of [12], we shall now characterize those measures in $\mathscr{M}_{0}$ that give rise to a densely defined form $\mathfrak{h}+\mu$.

\subsection{Theorem. Let $\mu \in \mathscr{M}_{0}$. Then the following conditions are equivalent:}

(i) $\mathbf{D}(\mathfrak{h}+\mu)$ is dense in $L_{2}(\mathbf{X}, \mathbf{m})$.

(ii) For each $\varepsilon>0$ there exist a closed set $A \subset \mathbf{X}$ and an open set $U \subset \mathbf{X}$ such that $\mathbf{m}(A)=0, \operatorname{Cap}(U)<\varepsilon$, and $\chi_{\mathbf{X} \backslash U} \cdot \mu$ is locally finite on $\mathbf{X} \backslash A$.

(iii) For each $\varepsilon>0$ there exist a closed set $A \subset \mathbf{X}, f \in \mathbf{D}$, and $\varphi \in[f]_{q}$, such that $\mathbf{m}(A)=0,0 \leq f \leq 1,\|f\|_{\mathfrak{h}} \leq \varepsilon$, and $(1-\varphi) \mu$ is locally finite on $\mathbf{X} \backslash A$.

The proof of [12, Theorem 1.6, p. 144] can be modified to cover the above case. An analogous modification is worked out in the proof of Theorem 2.2.

\section{SMOOTH MEASURES}

Let us call a sequence $\left(F_{n}\right)_{n \in \mathbb{N}}$ of closed sets a local nest (for $\mathfrak{h}$ ) if, for every compact $K \subset \mathbf{X}$,

$$
\operatorname{Cap}\left(K \backslash F_{n}\right) \rightarrow 0 \text { for } n \rightarrow \infty .
$$

Remark. Let $\left(F_{n}\right)_{n \in \mathbb{N}}$ be a local nest, $\mu \in \mathscr{M}_{0}$. Then

$$
\mu\left(\mathbf{X} \backslash \bigcup_{n \in \mathbb{N}} F_{n}\right)=0 .
$$

Proof. Fix an increasing sequence $\left(\mathbf{X}_{k}\right)$ of compact subsets of $\mathbf{X}$, such that $\bigcup \stackrel{\circ}{\mathbf{X}}_{k}=\mathbf{X}$. For every $k \in \mathbb{N}, \operatorname{Cap}\left(\mathbf{X}_{k} \backslash \bigcup_{n \in \mathbb{N}} F_{n}\right) \leq \lim _{n \rightarrow \infty} \operatorname{Cap}\left(\mathbf{X}_{k} \backslash F_{n}\right)=0$. Consequently, $\mu\left(\mathbf{X}_{k} \backslash \bigcup_{n \in \mathbb{N}} F_{n}\right)=0$. Together with $\mathbf{X}=\bigcup_{k \in \mathbb{N}} \mathbf{X}_{k}$, this proves the assertion.

A measure $\mu$ is called smooth (cf. [9, p. 72]) if it charges no set of zero capacity and there exists a local nest $\left(F_{n}\right)_{n \in \mathbb{N}}$ consisting of $\mu$-integrable sets. By

$$
\mathscr{S}:=\mathscr{S}(\mathfrak{h}) \text {, }
$$

we denote the set of smooth measures.

The following density theorem proves the usefulness of local nests.

2.1. Theorem. Let $\left(F_{n}\right)_{n \in \mathbb{N}}$ be a local nest. Then

$$
\left\{f \in \mathbf{D} ; 0 \leq \tilde{f} \leq \chi_{F_{n}} \text { for some } n \in \mathbb{N}\right\}
$$

is a total set in $\left(\mathbf{D},(\cdot \cdot \cdot)_{\mathfrak{h}}\right)$.

Proof. Let $\varphi \in \mathbf{D} \cap \mathbf{C}_{c}(\mathbf{X})$. Since $\mathfrak{h}$ is regular and $L:=\operatorname{lin}\{\ldots\}$ (the linear hull of the set that appears in the statement of the theorem) is convex, it is enough to construct a sequence $\left(\varphi_{n}\right)_{n \in \mathbb{N}}$ in $L$ that converges to $\varphi$ in the weak topology 
of $\left(\mathbf{D},(\cdot \mid \cdot)_{\mathfrak{h}}\right)$. Since $K:=\operatorname{supp} \varphi$ is compact, $\operatorname{Cap}\left(K \backslash F_{n}\right) \rightarrow 0$ for $n \rightarrow \infty$. Hence, we find quasi-continuous $\psi_{n} \in \mathbf{D}, \chi_{K \backslash F_{n}} \leq \psi_{n} \leq 1$ for $n \in \mathbb{N}$ such that $\psi_{n} \rightarrow 0$ in $\left(\mathbf{D},(\cdot \mid \cdot)_{\mathfrak{h}}\right)$. For $n \in \mathbb{N}, \varphi_{n}:=\varphi\left(1-\psi_{n}\right)$, it follows that $\varphi_{n} \in \mathbf{D}$ and

$$
\left\|\varphi_{n}\right\|_{\mathfrak{h}} \leq\|\varphi\|_{\mathfrak{h}}+\left\|\varphi \psi_{n}\right\|_{\mathfrak{h}} \leq\|\varphi\|_{\mathfrak{h}}+\|\varphi\|_{\infty}\left\|\psi_{n}\right\|_{\mathfrak{h}}+\|\varphi\|_{\mathfrak{h}}\left\|\psi_{n}\right\|_{\infty}
$$

(see [9, Theorem 1.4.2(ii), p. 25]). Therefore, $\left(\varphi_{n}\right)_{n \in \mathbb{N}}$ is bounded in $\mathbf{D}$. Moreover, for every $\psi \in \mathbf{D}(H)$,

$$
\mathfrak{h}\left[\varphi_{n}, \psi\right]=\left(\varphi_{n} \mid H \psi\right) \rightarrow(\varphi \mid H \psi)=\mathfrak{h}[\varphi, \psi],
$$

since $\varphi_{n} \rightarrow \varphi$ in $\mathbf{L}_{2}(\mathbf{X}, \mathbf{m})$. This implies the weak convergence of $\left(\varphi_{n}\right)$; by construction, $\varphi_{n} \in L$ for each $n \in \mathbb{N}$.

With the last theorem at hand we can now prove the main result of this article. It contains an operator theoretic description of $\mathscr{S}$ that, in particular, gives the following answer to a question raised in [3, Remark, p. 6]: For the potential $V$ constructed in [13], $V d x$ is smooth.

2.2. Theorem. Let $\mu \in \mathscr{M}_{0}$. Then the following conditions are equivalent:

(i) $\mu$ is smooth.

(ii) $\mathbf{D}(\mathfrak{h}+\mu)$ is dense in $\left(\mathbf{D},(\cdot \mid \cdot)_{\mathfrak{h}}\right)$.

(iii) In the strong resolvent sense, $\mathfrak{h}+a \mu \rightarrow \mathfrak{h}$ for $a \downarrow 0$.

Remark. For $\mu \in \mathscr{M}_{0}$ and $a>0$ it follows that $a \mu \in \mathscr{M}_{0}$ so that the condition in (iii) makes sense.

Proof of Theorem 2.2. (i) $\Rightarrow$ (ii) follows from Theorem 2.1 since

$$
\left\{f \in \mathbf{D} ; 0 \leq \tilde{f} \leq \chi_{F_{n}} \text { for some } n \in \mathbb{N}\right\} \subset \mathbf{D}(\mathfrak{h}+\mu)
$$

whenever $\left(F_{n}\right)_{n \in \mathbb{N}}$ is a local nest consisting of $\mu$-integrable sets.

(ii) $\Rightarrow$ (i) (cf. [12, Proof of Theorem 1.5, p. 143]). Let $\left(\mathbf{X}_{k}\right)$ be as in the proof of the remark. Since $\mathfrak{h}$ is regular, there exist functions $\psi_{n} \in \mathbf{D} \cap \mathbf{C}_{c}(\mathbf{X})$, $\psi_{n} \geq \chi \mathbf{x}_{n}$ for every $n \in \mathbb{N}$. By assumption there is a sequence $\left(f_{n}\right)_{n \in \mathbb{N}}$ in $\mathbf{D}(\mathfrak{h}+\mu)$ satisfying $f_{n} \leq \psi_{n}$ for $n \in \mathbb{N}$ and $\left\|\varphi_{n}-\psi_{n}\right\|_{\mathfrak{h}} \rightarrow 0$ for $n \rightarrow \infty$. According to [9, Theorem 3.1.3] there is an open set $U_{n} \subset \mathbf{X}$ and a quasicontinuous $\varphi_{n} \in\left[f_{n}\right]_{q}$ such that $\operatorname{Cap}\left(U_{n}\right) \leq 2^{-n}$ and $\varphi_{n} / U_{n}$ is continuous. Let

$$
F_{n}:=\left(X_{n} \backslash U_{n}\right) \cap\left\{y \in \mathbf{X} ; \varphi_{n}(y) \geq n^{-1}\right\}
$$

for $n \in \mathbb{N}$. Since $D_{n}:=\left\{y \in \mathbf{X} ; \varphi_{n}(y) \geq n^{-1}\right\}$ is relatively closed in $X_{n} \backslash U_{n}$, $F_{n}$ is compact for every $n \in \mathbb{N} . F_{n} \subset D_{n}$ implies that $\mu\left(F_{n}\right)<\infty$ for every $n \in \mathbb{N}$. Let $K \subset X$ be compact. Then $K \subset X_{n}$ for $n$ large enough. It follows that

$$
K \backslash F_{n} \subset U_{n} \cup\left(D_{n} \cap\left(X_{n} \backslash U_{n}\right)\right),
$$

and since $\operatorname{Cap}\left(U_{n}\right) \rightarrow 0$, it remains to show

$$
\operatorname{Cap}\left(D_{n} \cap\left(X_{n} \backslash U_{n}\right)\right) \rightarrow 0 .
$$

To verify this, observe that

$$
0 \leq\left(1-n^{-1}\right) \chi_{D_{n} \cap\left(X_{n} \backslash U_{n}\right)} \leq \psi_{n}-\varphi_{n},
$$

which yields

$$
\operatorname{Cap}\left(D_{n} \cap\left(X_{n} \backslash U_{n}\right)\right) \leq\left(1-n^{-1}\right)^{-2}\left\|\varphi_{n}-\psi_{n}\right\|_{\mathfrak{h}}^{2} \rightarrow 0,
$$


where we used $[9$, Lemma 3.1 .5$, p. 66] for the last inequality.

(ii) $\Leftrightarrow$ (iii). By [11, Theorem 3.2, p. 382f], h $+a \mu \rightarrow(\mathfrak{h} \mid \mathbf{D}(\mathfrak{h}+\mu))_{r}^{-}$, where the last symbol stands for the closure of the regular part (see [11]) of $\mathfrak{h} \mid \mathbf{D}(\mathfrak{h}+\mu)$, which simply equals the closure of $\mathfrak{h} \mid \mathbf{D}(\mathfrak{h}+\mu)$.

In our point of view, the equivalent conditions (ii) and (iii) in the above theorem are very natural. In terms of the underlying process, they can be interpreted as follows: $\mu$ is not smooth iff "too many particles" are totally absorbed by $\mu$, which forces elements of $\mathbf{D}(\mathfrak{h}+\mu)$ to vanish on some set.

To present a typical example, let us take $V(x)=\left|x_{1}\right|^{-1}$ and $\mu=V d x$ in the situation of Example 2. Then $\mathbf{D}(\mathfrak{h}+\mu)=\stackrel{\circ}{W} \underset{2}{1}\left(\mathbb{R}^{\nu} \backslash\{0\} \times \mathbb{R}^{\nu-1}\right)$, i.e., the limit of $\mathfrak{h}+a \mu$ as $a \downarrow 0$ is the form corresponding to the Dirichlet-Laplacian on $\mathbb{R}^{\nu} \backslash\{0\} \times \mathbb{R}^{\nu-1}$. This means that typical Brownian particles cannot pass the barrier that is given by $V$.

\section{ACKNOWLEDGMENT}

Concluding this paper, I want to thank Professor J. Voigt very warmly for his help and encouragement. Moreover, it is a pleasure to acknowledge fruitful discussions with Dr. T. Sturm.

\section{REFERENCES}

1. S. Albeverio, J. Brasche, and M. Röckner, Dirichlet forms and generalized Schrödinger Operators, Schrödinger Operators (H. Holden and A. Jensen, eds.), Proceedings, Sonderborg, Lecture Notes in Math., Springer-Verlag, Berlin and New York.

2. S. Albeverio and Zhiming Ma, Perturbation of Dirichlet forms, SFB 237 preprint 1989; J. Funct. Anal. 99 (1991), 332-356.

3. __ Nowhere Radon smooth measures, perturbation of Dirichlet forms and singular quadratic forms, BiBoS preprint 352, 1989; (N. Christopeit et al., eds.), Proceedings, Bad Honnef 1988, Lecture Notes Control Inf., vol. 126, Springer-Verlag, New York, 1989.

4. __ Additive functionals, nowhere Radon and Kato class smooth measures associated with Dirichlet forms, SFB 237 preprint, 1989.

5. J. Baxter, G. DalMaso, and U. Mosco, Stopping times and $\Gamma$-convergence, Trans. Amer. Math. Soc. 303 (1987), 1-38.

6. Ph. Blanchard and Zhiming Ma, Semigroup of Schrödinger operators with potentials given by Radon measures, Stochastic Processes-Physics and Geometry (S. Albeverio et al., eds.), Proceedings 2nd. Internat. Conf. Adcona-Como-Locarno 1988, World Sci., Singapore, 1989.

7. __ Smooth measures and Schrödinger semigroups, BiBoS preprint 295, 1987.

8. __ New results on the Schrödinger semigroups with potentials given by signed smooth measures, in "Proceedings Siliviri Workshop 1988" (Korezioglu et al., eds.), Lecture Notes in Math., vol. 1444, Springer-Verlag, New York and Berlin, 1990.

9. M. Fukushima, Dirichlet forms and Markov processes, North Holland, Amsterdam, 1980.

10. T. Kato, Perturbation theory for linear operators, 2nd ed., Springer-Verlag, Berlin, 1980.

11. B. Simon, A canonical decomposition for quadratic forms with applications to monotone convergence theorems, J. Funct. Anal. 28 (1978), 377-385.

12. P. Stollmann, Admissible and regular potentials for Schrödinger forms, J. Operator Theory 18 (1987), 139-151.

13. P. Stollmann and J. Voigt, A regular potential which is nowhere in $L_{1}$, Lett. Math. Phys. 9 (1985), 227-230.

14. T. Sturm, Schrödinger equations with discontinuous solutions, Potential Theory (Prague 1987), Plenum Press, New York and London, 1988, pp. 333-337. 
15. __ Störung von Hunt-Prozessen durch signierte additive Funktionale, Dissertation, Erlangen, 1988.

16. J. Voigt, Absorption semigroups, their generators, and Schrödinger semigroups, J. Funct. Anal. 67 (1986), 167-205.

Fachbereich Mathematik der Universität Oldenburg, Ammerländer Heerstasse 114118, D-2900 Oldenburg, Germany

Current address: Fachbereich Mathematik, Johann Wolfgang Goethe-Universität, Robert-Mayer Str. 10, D-6000 Frankfurt, Germany 